\title{
Nobiletin Prevents Body Weight Gain and Bone Loss in Ovariectomized C57BL/6J Mice
}

\author{
Young-Sil Lee ${ }^{1}$, Midori Asai', Sun-Sil Choi ${ }^{1}$, Takayuki Yonezawa', Toshiaki Teruya ${ }^{2}$, \\ Kazuo Nagai ${ }^{1}$, Je-Tae Woo ${ }^{3,4}$, Byung-Yoon Cha ${ }^{*}$ \\ ${ }^{1}$ Research Institute for Biological Functions, Chubu University, Kasugai, Japan \\ ${ }^{2}$ Faculty of Education, University of the Ryukyus, Nishihara, Japan \\ ${ }^{3}$ Department of Biological Chemistry, Chubu University, Kasugai, Japan \\ ${ }^{4}$ Department of Research and Development, Erina Co., Inc., Tokyo, Japan \\ Email: ${ }^{\text {bycha@isc.chubu.ac.jp }}$
}

Received 10 August 2014; revised 8 September 2014; accepted 19 September 2014

Copyright (C) 2014 by authors and Scientific Research Publishing Inc.

This work is licensed under the Creative Commons Attribution International License (CC BY). http://creativecommons.org/licenses/by/4.0/

(c) (i) Open Access

\begin{abstract}
Obesity and osteoporosis are associated with estrogen deficiency following menopause. Therefore, it is important to prevent and treat both disorders to maintain a healthy life in postmenopausal women. Nobiletin, a polymethoxylated flavone, exhibits various pharmacologic effects, including anti-tumor and anti-inflammatory activities. Therefore, in this study, we examined the effects of nobiletin on obesity, obesity-related metabolic disorders, and bone mass in ovariectomized (OVX) mice. Mice were divided into four groups and underwent sham operation or OVX. OVX mice were treated with 50 or $100 \mathrm{mg} / \mathrm{kg}$ nobiletin, or received vehicle alone $(0.3 \%$ carboxyl methyl cellulose $/ 0.5 \%$ dimethyl sulfoxide). Nobiletin decreased body weight gain and white adipose tissue weight in OVX mice. Nobiletin also decreased triglyceride levels, and tended to reduce plasma total cholesterol and glucose levels. Additionally, nobiletin prevented the reduction in bone mineral density of the trabecular region of the femur in OVX mice. Taken together, our results suggest that nobiletin improves adiposity, dyslipidemia, hyperglycemia, and prevents bone loss in OVX mice. Therefore, nobiletin is expected to have beneficial effects for the prevention and improvement of metabolic disorders and osteoporosis in postmenopausal women.
\end{abstract}

\section{Keywords}

Nobiletin, Ovariectomy, Obesity, Lipid and Glucose Metabolism, Bone Mineral Density

\footnotetext{
${ }^{*}$ Corresponding author.
} 


\section{Introduction}

Estrogen is an important factor for protection against obesity in females. Estrogen deficiency leads to osteoporosis, as well as body weight gain [1] [2]. Recent studies have shown that postmenopausal women have greater body fat and visceral fat compared with premenopausal women [1]-[4]. Obesity is associated with several metabolic disorders including dyslipidemia, insulin resistance, and cardiovascular disease [1] [2]. Because osteoporosis and obesity are major health problems in postmenopausal women, it is important to identify strategies to prevent or treat these disorders and maintain a healthy life in postmenopausal women.

Natural phytoestrogens are increasingly being used to prevent or improve metabolic disorders, and are thought to reduce the risk of osteoporosis in postmenopausal women [5] [6]. Additionally, phytoestrogens seem to lack the undesirable side effects associated with estrogen. Therefore, there is growing interest in using natural compounds to prevent and improve metabolic disorders and osteoporosis in postmenopausal women.

Nobiletin is a polymethoxylated flavone present in some citrus fruits such as Citrus depressa (shiikuwasa) and Citrus sinensis (orangens) [7] [8]. Nobiletin was reported to exhibit biological effects via its anti-inflammatory, anti-tumor, and neuroprotective properties [9]-[11]. It was also recently reported that nobiletin can regulate bone metabolism by inhibiting osteoclast formation and bone resorption induced by interleukin (IL)-1 in osteoblasts, and preventing bone loss in OVX mice [12]. Recent reports have also revealed that nobiletin may be able to regulate lipid metabolism. Nobiletin enhances lipolysis and suppresses adipogenesis, although it is also reported that nobiletin induces adipocyte differentiation [13]-[15]. Our previous studies revealed that nobiletin reduces adiposity, plasma triglyceride (TG) levels, and insulin resistance in high-fat diet (HFD)-induced obese mice [16]. In the present study, we investigated the effects of nobiletin on obesity and bone mass in OVX mice.

\section{Materials and Methods}

\subsection{Isolation of Nobiletin}

Nobiletin was isolated and identified as described in our previous report [16].

\subsection{Animals and Experimental Design}

Female C57BL/6J mice were purchased from Japan SLC (Shizuoka, Japan) at 6 weeks of age. The mice were housed under temperature- $\left(23^{\circ} \mathrm{C} \pm 3^{\circ} \mathrm{C}\right)$ and humidity-controlled conditions with a 12-h light/dark cycle, and were given free access to food and water throughout the experiment. After acclimatization for 1 week with a standard rodent normal-fat diet (CRF-1; Charles River, Japan), the mice underwent either sham-operation (sham, $n=8$ ) or ovariectomy (OVX, $n=24)$. After surgery, mice were allowed to recover under normal conditions. Two days later, the OVX mice were randomly divided into three groups ( $n=8$ mice/group) and treated with 50 $(\mathrm{OVX}+50 \mathrm{NOB})$ or $100(\mathrm{OVX}+100 \mathrm{NOB}) \mathrm{mg} / \mathrm{kg}$ nobiletin, or vehicle (OVX control group). The vehicle was $0.3 \%$ carboxyl methyl cellulose $/ 0.5 \%$ dimethyl sulfoxide. Nobiletin and vehicle were administered by oral gavage once daily for 12 weeks. Mice in the sham control group were administered with vehicle alone. Body weight and food intake for each mouse was measured two times per week during the study. The study was approved by The Animal Experimental Committee of Chubu University, and the mice were maintained in accordance with their guidelines.

\subsection{Plasma, Tissue, and Bone Sampling}

At the end of the 12-week study, the mice were anesthetized with a high dose of ether. Plasma samples were obtained by centrifuging blood samples at $5000 \times g$ for $15 \mathrm{~min}$ at $4^{\circ} \mathrm{C}$. The resulting plasma samples were stored at $-80^{\circ} \mathrm{C}$ until analysis. Liver, white adipose tissues (WAT; reproductive, perirenal, and mesenteric WAT), and the uterus were immediately excised, rinsed, and weighed. The femurs were also excised, soft tissue was carefully removed from the bone without damaging trabecular tissue, and the femoral bones were fixed in $70 \%$ ethanol.

\subsection{Plasma Biochemistry}

Plasma total cholesterol (T-CHO), TG, and glucose levels were determined using commercially available enzyme assay kits (Cholesterol E-Test, Triglyceride E-Test, and Glucose C II-Test, respectively; Wako Pure Chemical Industries, Osaka, Japan) according to the manufacturer's protocols. 


\subsection{Peripheral Quantitative Computed Tomography (pQCT) Analysis}

Isolated bones were measured by pQCT (XCT Research, SA+, Stratec Medizintechnik GmbH, Pforzheim, Germany) with a tube voltage of $50.5 \mathrm{kV}$ and a tube current of $0.281 \mathrm{~mA}$. The scan speed was $5 \mathrm{~mm} / \mathrm{s}$ with a voxel resolution of $0.07 \mathrm{~mm}$. The analytical parameters for cortical bone mineral density (BMD) were set as a threshold of $690 \mathrm{mg} / \mathrm{cm}^{3}$ and a peel mode of 20. Trabecular BMD was $<395 \mathrm{mg} / \mathrm{cm}^{3}$ with a peel mode of 20 . A femur slice located $0.6 \mathrm{~mm}$ from the distal end of the growth plate was used to measure trabecular and cortical BMD. Trabecular bone was defined by setting an internal area of 35\% of the total cross-sectional area. Total BMD, trabecular BMD, and cortical BMD were calculated using pQCT software (Makejob; StratecMedizintechnik GmbH).

\subsection{Statistical Analysis}

Data are expressed as means \pm standard error of the mean. Differences in mean values between each group were analyzed by one-way analysis of variance, followed by Dunnett's test. Values of $p<0.05$ were considered to indicate statistical significance. All analyses were conducted using IBM-SPSS version 20 (IBM, New York, NY, USA).

\section{Results}

\subsection{Effects of Nobiletin on Body Weight Gain and Food Intake}

Body weight gain and food intake are shown in Figure 1. Body weight gain was significantly greater in the OVX group than in the sham group $(p<0.005)$. Body weight gain was significantly lower in the OVX + $100 \mathrm{NOB}$ group than in the OVX group $(p<0.05)$ but was not significantly differentbetween the OVX $+50 \mathrm{NOB}$ and OVX groups. Food intake was comparable among all four groups.

\subsection{Effects of Nobiletin on Organ Weight}

Organ weight is shown in Figure 2. Uterus weight was significantly lower in the OVX group than in the sham group ( $p<0.005)$, indicating that the mice were estrogen deficient. Uterus weight tended to be higher in the OVX +100 NOB group than in the OVX group, although did not significantly. Liver weight did not differ among the four groups. WAT weight was significantly higher in the OVX group compared with the sham group $(p<0.005)$. The reproductive, perirenal, and total WAT weights were significantly lower in the OVX + 100NOB group than in the OVX group (all, $p<0.05$ ). However, there was no difference in WAT weights between the OVX + 50NOB group and the OVX group.

\subsection{Effects of Nobiletin on Plasma Biochemistry}

Figure 3 shows the effects of nobiletin on plasma biochemistry. Plasma T-CHO levels were significantly higher in the OVX group than in the shamgroup $(p<0.05)$. Plasma T-CHO levels were decreased in the OVX + 100NOB group compared with the OVX group, although not significantly. Plasma TG levels were significantly

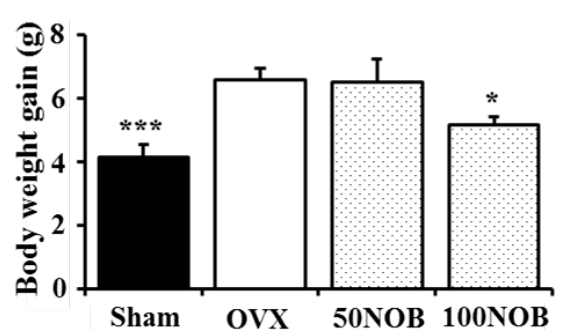

(a)

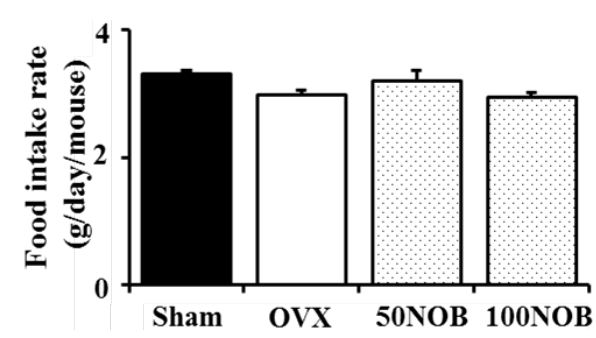

(b)

Figure 1. Effects of nobiletin on body weight gain (a) and food intake (b). Sham: shamoperated mice; OVX: ovariectomized mice; 50NOB: OVX + $50 \mathrm{mg} / \mathrm{kg}$ nobiletin; $100 \mathrm{NOB}$ : OVX $+100 \mathrm{mg} / \mathrm{kg}$ nobiletin. Values are means \pm standard error of the mean $(n=8$ mice/ group). ${ }^{*} p<0.05$ and ${ }^{* * *} p<0.005$ vs the OVX group. 


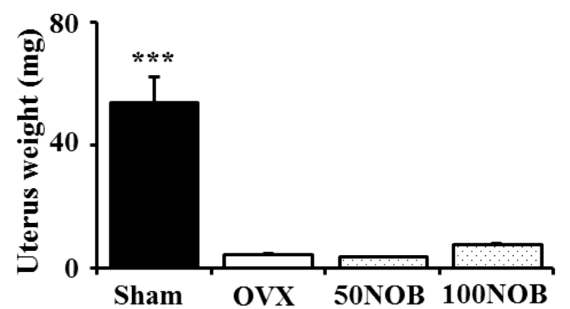

(a)

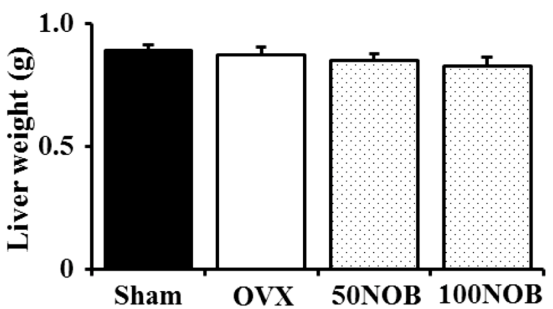

(b)

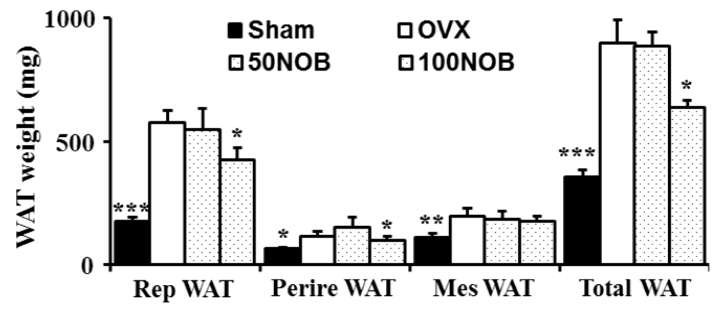

(c)

Figure 2. Effects of nobiletin onuterus weight (a), liver weight (b) and white adipose tissue (WAT) weight (c). RepW: reproductive WAT; PeriW: perirenal WAT; MesW: mesenteric WAT; TotalW: total WAT weight; Sham: sham-operated mice; OVX: ovariectomized mice; 50NOB: OVX $+50 \mathrm{mg} / \mathrm{kg}$ nobiletin; 100NOB: OVX $+100 \mathrm{mg} / \mathrm{kg}$ nobiletin. Values are means \pm standard error of the mean ( $n=8$ mice/group). ${ }^{*} p<0.05,{ }^{*} p<0.01$, and ${ }^{* * *} p<$ 0.005 vs the OVX group.

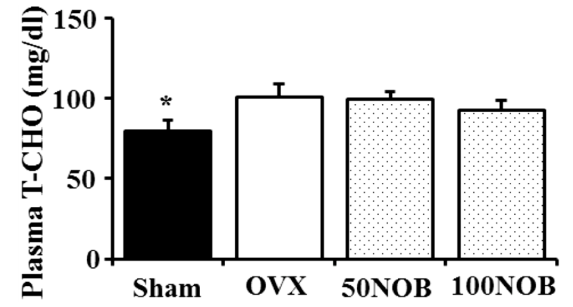

(a)

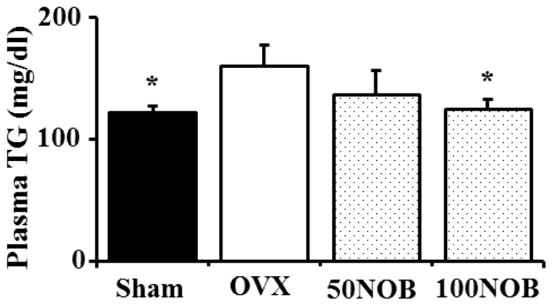

(b)

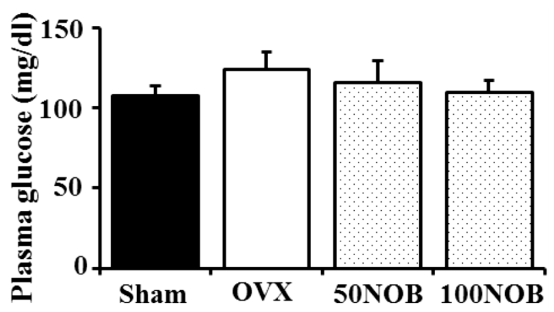

(c)

Figure 3. Effects of nobiletin on plasma total cholesterol (T-CHO; (a)), triglyceride (TG; (b)), and glucose (c) levels. Sham: sham-operated mice; OVX: ovariectomized mice; 50NOB: OVX+50 mg/kg nobiletin; 100NOB: OVX $+100 \mathrm{mg} / \mathrm{kg}$ nobiletin. Values are means \pm standard error of the mean ( $n=8$ mice/group). ${ }^{*} p<0.05$ vs the OVX group.

higher in the OVX group than in the sham group $(p<0.05)$. Plasma TG levels were lower in the OVX + $100 \mathrm{NOB}$ group, but not in the OVX + 50NOB group, compared with the OVX group. Plasma glucose levels did not differ between the sham and OVX groups, although they tended to be lower in the OVX $+100 \mathrm{NOB}$ group than in the OVX group.

\subsection{Effects of Nobiletin on BMD}

Figure 4 shows the effects of nobiletin on BMD in OVX rats. Total femoral BMD and trabecular BMD were 


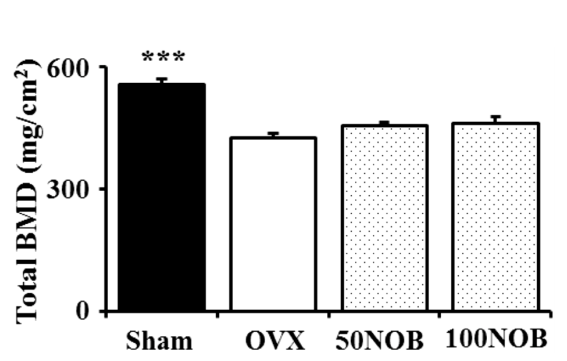

(a)

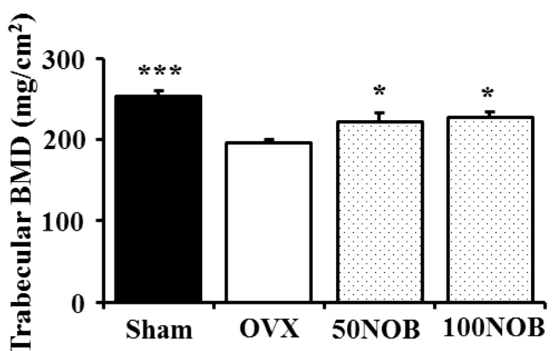

(b)

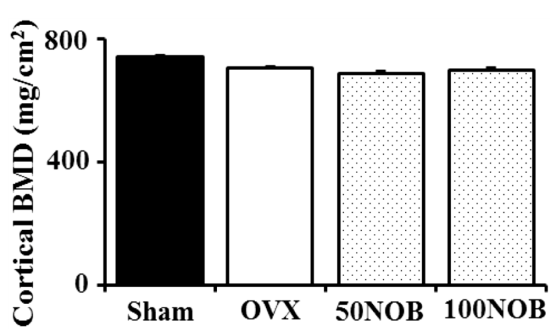

(c)

Figure 4. Effects of nobiletin on total femoral bone mineral density (BMD; (a)), trabecular BMD (b), and cortical BMD (c). Sham: sham-operated mice; OVX: ovariectomized mice; 50NOB: OVX + $50 \mathrm{mg} / \mathrm{kg}$ nobiletin; 100NOB: OVX + $100 \mathrm{mg} / \mathrm{kg}$ nobiletin. NFD: normal-fat diet; HFD: high-fat diet. Values are means \pm standard error of the mean $(n=8) .{ }^{*} p$ $<0.05$ and ${ }^{* * *} p<0.005$ vs the OVX group.

significantly lower in the OVX group than in the sham group (both, $p<0.005$ ). The decrease in total femoral BMD caused by OVX was attenuated by both doses of nobiletin, although this not significantly. Trabecular BMD was significantly greater in both OVX + NOB groups than in the OVX group (both, $p<0.05$ ). Cortical BMD did not differ among the four groups.

\section{Discussion}

In the present study, we examined whether nobiletin could reduce obesity, obesity-related metabolic disorders, and osteoporosis in OVX mice. To our knowledge, the present study is the first to show that nobiletin prevents the increases in body weight, WAT weight, and plasma TG, as well as bone loss, in OVX mice.

In the present study, nobiletin reduced increases in body weight gain and WAT weight in OVX mice. It has been reported that estrogen is capable of preventing obesity in females. OVX mice are characterized by increased food intake and decreased energy expenditure, which lead to obesity [17]. It was reported that treating OVX mice with estradiol prevented the development of obesity [18].

In the present study, food intake was similar in the sham and OVX groups, and was not affected by nobiletin. Therefore, the reduction in body weight gain and WAT weight in this study were not caused by changes in food intake. Recent studies and our own in vivo data indicate that nobiletin regulates adipogenesis and lipolysis. For example, nobiletin enhances lipolysis in differentiated adipocytes by activating the cAMP-cAMP response element-binding pathway and suppresses lipid accumulation by downregulating peroxisome proliferator-activated receptor (PPAR) $\gamma$, and activating AMP-activated protein kinase. However, it was reported that nobiletincan induce adipocyte differentiation [13]-[15]. Furthermore, we previous reported that nobiletin increased the expression of energy expenditure-related genes, such as PPAR $\alpha$ and carnitine palmitoyltransferase I, in HFD-induced obese mice [16]. Based on these earlier findings, it is likely that increased lipolysis and energy expenditure may be involved in the reduced body weight gain and WAT weight in nobiletin-treated mice. Further studies are needed to examine the effects of nobiletin on the expression of lipid metabolism-related genes.

Obesity-related metabolic disorders, such as hyperlipidemia, hyperglycemia, and glucose intolerance, are significant problems in postmenopausal women. In the present study, nobiletinreduced plasma TG levels and tended to reduce plasma T-CHO and glucose levels in OVX mice. In our previous study, we showed that nobiletin improved hypertriglyceridemia [16]. These results suggest that nobiletin may improve obesity-related meta- 
bolic disorders, such as hyperlipidemia, in postmenopausal women.

Osteoporosis is a skeletal disease characterized by a reduction in bone strength, increasing the risk of fracture. Osteoporosis in postmenopausal women is caused by a decrease in estrogen levels and an increase in bone resorption [19]. In our present study, we showed that nobiletin inhibited the decrease in trabecular BMD of OVX mice and showed tendency to increase total femoral BMD in OVX mice. Previous studies have shown that nobiletin suppresses osteoclast formation and bone resorption by inhibiting nuclear factor-kB-dependent transcription and prostaglandin E production in osteoblasts via the activity of IL-1. This report also showed that nobiletin prevents bone loss in OVX mice [12] (Harada et al. 2011). Based on our results and this earlier report, nobiletin is expected to prevent osteoporosis in postmenopausal women.

\section{Conclusion}

In conclusion, treatment with nobiletin decreased body weight gain, WAT weight, and plasma TG levels in OVX mice. Nobiletin tended to decrease plasma T-CHO levels and glucose levels in OVX mice, and prevented the decrease in BMD following OVX. These results suggest that nobiletin may improve adiposity, hypertriglyceridemia, and bone metabolism in OVX mice, as a model of the postmenopausal state. Therefore, nobiletin may have beneficial effects for the prevention and treatment of metabolic disorders and osteoporosis in postmenopausal women.

\section{References}

[1] You, T., Ryan, A.S. and Nicklas, B.J. (2004) The Metabolic Syndrome in Obese Postmenopausal Women: Relationship to Body Composition, Visceral Fat, and Inflammation. The Journal of Clinical Endocrinology Metabolism, 89, 5517-5522.

http://press.endocrine.org/doi/abs/10.1210/jc.2004-0480?url_ver=Z39.88-2003\&rfr_id=ori:rid:crossref.org\&rfr_dat=cr pub=pubmed\&

http://dx.doi.org/10.1210/jc.2004-0480

[2] Kaaja, R.J. (2008) Metabolic Syndrome and the Menopause. Menopause International, 14, 21-25.

http://min.sagepub.com/content/14/1/21.abstract http://dx.doi.org/10.1258/mi.2007.007032

[3] Cooke, P.S. and Naaz, A. (2004) Role of Estrogens in Adipocyte Development and Function. Experimental Biology and Medicine (Maywood), 229, 1127-1235. http://ebm.sagepub.com/content/229/11/1127.abstract

[4] Lobe, R.A. (2008) Metabolic Syndrome after Menopause and the Role of Hormones. Maturitas, 60, 10-18. http://www.maturitas.org/article/S0378-5122(08)00039-X/abstract http://dx.doi.org/10.1016/j.maturitas.2008.02.008

[5] Anderson, J.W., Johnstone, B.M. and Cook-Newell, M.E. (1995) Meta-Analysis of the Effects of Soy Protein Intake on Serum Lipids. The New England Journal of Medicine, 333, 276-282.

http://www.nejm.org/doi/full/10.1056/NEJM199508033330502 http://dx.doi.org/10.1056/NEJM199508033330502

[6] Messina, M.J. (2002) Soy Foods and Soybean Isoflavones and Menopausal Health. Nutrition in Clinical Care, 5, $272-$ 282. http://onlinelibrary.wiley.com/doi/10.1046/j.1523-5408.2002.05602.x/abstract http://dx.doi.org/10.1046/j.1523-5408.2002.05602.x

[7] Mercader, J., Wanecq, E., Chen, J. and Carpéné, C. (2011) Isopropylnorsynephrine Is a Stronger Lipolytic Agent in Human Adipocytes than Synephrine and Other Amines Present in Citrus Aurantium. Journal of Physiology and Biochemistry, 67, 443-452. http://link.springer.com/article/10.1007\%2Fs13105-011-0078-2

[8] Nagata, U., Sakamoto, K., Shiratsuchi, H., Ishi, T., Yano, M. and Ohta, H. (2006) Flavonoid Composition of Fruit Tissues of Citrus Species. Bioscience, Biotechnology, and Biochemistry, 70, 178-192. https://www.jstage.jst.go.jp/article/bbb/70/1/70_1_178/_article

[9] Murakami, A., Nakamura, Y., Torikai, K., et al. (2000) Inhibitory Effect of Citrus Nobiletin on Phorbol Ester-Induced Skin Inflammation Oxidative Stress, and Tumor Promotion in Mice. Cancer Research, 60, 5059-5066.

http://cancerres.aacrjournals.org/content/60/18/5059.long

[10] Silalahi, J. (2002) Anticancer and Health Protective Properties of Citrus Fruit Components. Asia Pacific Journal of Clinical Nutrition, 11, 79-84. http://onlinelibrary.wiley.com/doi/10.1046/j.1440-6047.2002.00271.x/abstract http://dx.doi.org/10.1046/j.1440-6047.2002.00271.x

[11] Nakajima, A., Yamakuni, T., Haraguchi, M., Omae, N., Song, S.Y., Kato, C., et al. (2007) Nobilein, a Citrus Flavonoid 
that Improves Memory Impairment, Rescues Bulbectomy-Induced Cholinergic Neurodegeneration in Mice. Journal of Pharmacological Sciences, 105, 122-126. https://www.jstage.jst.go.jp/article/jphs/105/1/105_1_122/_article

[12] Harada, S., Tominari, T., Matsumoto, C., Hirata, M., Takita, M., Inada, M. and Miyaura, C. (2011) Nobiletin, a Polymethoxy Flavonoid, Suppresses Bone Resorption by Inhibiting NF $\kappa$ B-Dependent Prostaglandin E Synthesis in Osteoblasts and Prevents Bone Loss Due to Estrogen Deficiency. Journal of Pharmacological Sciences, 115, 89-93. https://www.jstage.jst.go.jp/article/jphs/115/1/115_10193SC/_article http://dx.doi.org/10.1254/jphs.10193SC

[13] Saito, T., Abe, D. and Sekiya, K. (2007) Nobiletin Enhances Differentiation and Lipolysis of 3T3-L1 Adipocytes. Biochemical and Biophysical Research Communications, 357, 371-376. http://www.sciencedirect.com/science/article/pii/S0006291X07006109

[14] Kanda, K., Nishi, K., Kadota, A., Nishimoto, S., Liu, M.C. and Sugahara, T. (2011) Nobiletin Suppresses Adipocyte Differentiation of 3T3-L1 Cells by an Insulin and IBMX Mixture Induction. Biochimica et Biophysica Acta, 1820, 461-468. http://www.sciencedirect.com/science/article/pii/S0304416511002923

[15] Choi, Y., Kim, Y., Ham, H., Park, Y., Jeong, H.S. and Lee, J. (2011) Nobiletin Suppresses Adipogenesis by Regulating the Expression of Adipogenic Transcription Factors and the Activation of AMP-Activated Protein Kinase (AMPK). Journal of Agricultural and Food Chemistry, 59, 12843-12849. http://pubs.acs.org/doi/abs/10.1021/jf2033208 http://dx.doi.org/10.1021/jf2033208

[16] Lee, Y.S., Cha, B.Y., Saito, K., Yamakawa, H., Choi, S.S., Yamaguchi, K., et al. (2010) Nobiletin Improves Hyperglycemia and Insulin Resistance in Obese Diabetic ob/ob Mice. Biochemical Pharmacology, 79, 1674-1683. http://www.sciencedirect.com/science/article/pii/S0006295210000936 http://dx.doi.org/10.1016/j.bcp.2010.01.034

[17] Roger, N.H., Perfield II, J.W., Strissel, K.J., Obin, M.S. and Greenberg, A.S. (2009) Reduced Energy Expenditure and Increased Inflammation Are Early Events in the Development of Ovariectomy-Induced Obesity. Endocrinology, 150, 2161-2168.

http://press.endocrine.org/doi/abs/10.1210/en.2008-1405?url_ver=Z39.88-2003\&rfr_id=ori:rid:crossref.org\&rfr_dat=cr pub=pubmed http://dx.doi.org/10.1210/en.2008-1405

[18] Kolta, S., De Vernejoul, M.C., Meneton, P., Fechtenbaum, J. and Roux, C. (2003) Bone Mineral Measurements in Mice: Comparison of Two Devices. Journal of Clinical Densitometry, 6, 251-258. http://www.sciencedirect.com/science/article/pii/S109469500660276X http://dx.doi.org/10.1385/JCD:6:3:251

[19] Riggs, B.L. (1992) Osteoporosis. In: Wyngaarden, J.B., Ed., Textbook of Medicine, 19th Edition, Saunders, Philadelphia, 1426-1430. 
Scientific Research Publishing (SCIRP) is one of the largest Open Access journal publishers. It is currently publishing more than 200 open access, online, peer-reviewed journals covering a wide range of academic disciplines. SCIRP serves the worldwide academic communities and contributes to the progress and application of science with its publication.

Other selected journals from SCIRP are listed as below. Submit your manuscript to us via either submit@scirp.org or Online Submission Portal.
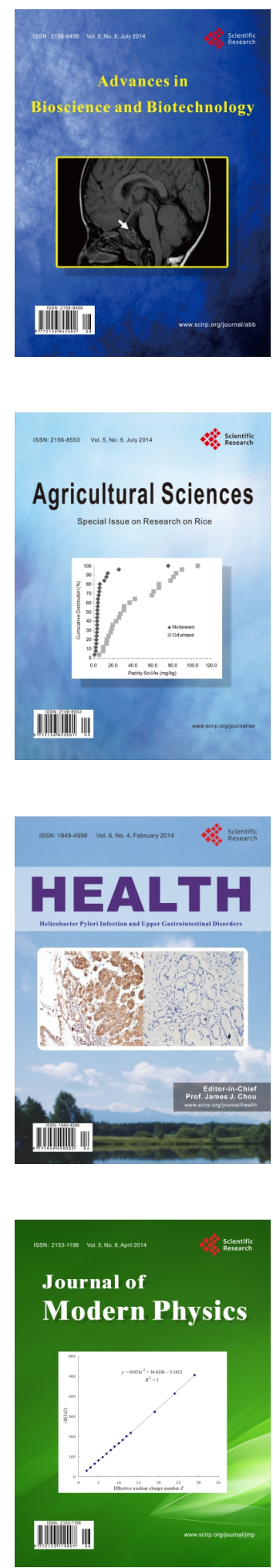
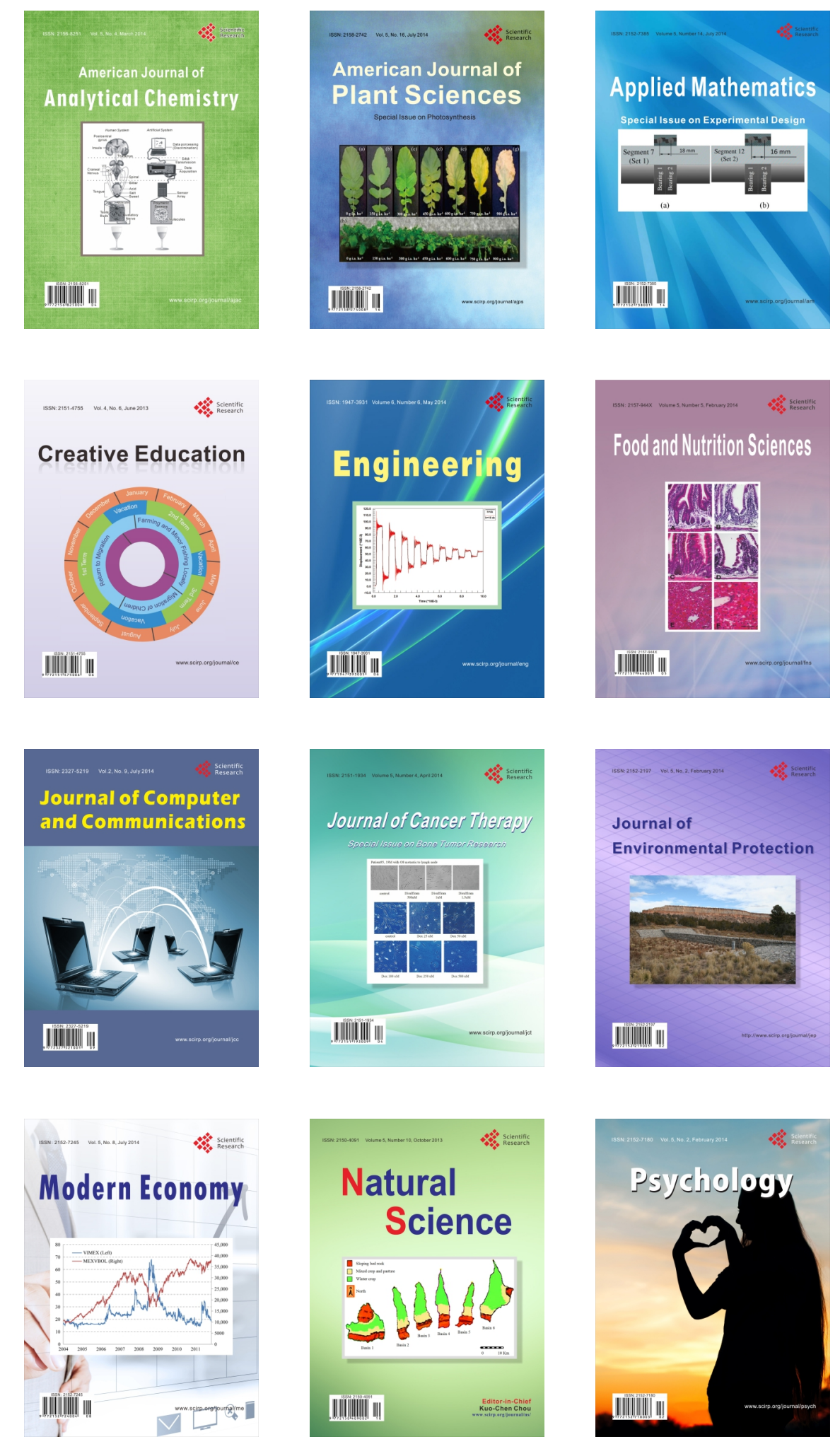